\title{
Programme basé sur Capprentissage inversé d'après le Youtube pour améliorer la production orale en situations de communication en français.
}

\section{Par : Tage Eldine Mohammed Eid}

Professeur adjoint de la méthodologie de FLE - Département de Curricula - Faculté de pédagogie - Université de Minia.

\section{Résumé}

Cette étude vise à utiliser un programme basé sur l'apprentissage inversé d'après le Youtube pour améliorer la production orale en situations de communication chez les étudiants de deuxième année au cycle secondaire. Pour ce faire, le chercheur a filmé le contenu oral de neuf modules en capsules vidéo à l'aide de deux étudiantes de quatrième année au département de français à la faculté de pédagogie ayant un niveau avancé ,puis, il a mis le contenu du programme à la disposition des apprenants en le téléchargeant sur une chaine de Youtube qu'il a déjà créée. Selon la stratégie de l'apprentissage inversé, les étudiants consultaient le contenu du programme à la maison et quand ils rentraient en classe, ils exécutaient des activités en présence du chercheur. En appliquant un test de la production orale de DELF A2, les résultats ont affirmé l'efficacité du programme sur le développement des compétences de la production orale chez les étudiants $d u$ groupe expérimental.

Mots clés : Programme - apprentissage - inversé - Youtube production - oral-situations - communication - français.

\section{INTRODUCTION}

A partir du 21e siècle, les technologies de l'information et de la communication en éducation (TICE) ont connu une évolution prépondérante. Les didacticiens des langues de leur rôle ont bien exploité ces technologies à développer l'apprentissage /enseignement des langues et surtout à l'ère de virus Covid-19. Cependant,les établissements scolaires 
et universitaires ont adapté la stratégie de l'apprentissage inversé en téléchargeant les cours sur les plateformes aux étudiants pendant la période du confinement.

L'école de Victoria aux Etats Unis organise des formations des enseignants d'anglais en ligne d'après l'apprentissage inversé. Ce type d'apprentissage appartient à la famille du "blended learning", car il mélange apprentissage en ligne et apprentissage en présentiel. Ce qui le rend spécifique. Cette méthode pédagogique vise à inverser la partie transmissive de savoir tandis que la partie d'activité se fait dans la salle de classe. Cette méthode peut être très utile à enseigner le français à l'aide des TICE en diffusant en ligne des capsules vidéos, des documents et des exercices à faire et en rentrant en salle de classe, l'enseignant guide les apprenants à pratiquer la langue en situations de communication.

Grâce à cette stratégie d'apprentissage, l'apprenant est au centre de son apprentissage et l'enseignant est le responsable de la construction de son propre savoir avec l'accompagnement de l'enseignant à distance et en présence.

Les médias sociaux numériques présentent un ensemble de services qui permettent de développer des conversations et des interactions sociales sur l'internet ou en situation de mobilité. Ces médias utilisent donc l'internet, afin de faciliter la création et le partage de publications, la collaboration et l'interaction sociale.

L'emploi des chaines de Youtube est un aspect important de l'application du numérique qui présente des 
activités variées convenables aux étudiants des établissements scolaires et universitaires. Beaucoup de chaines de Youtube ont été créées pour présenter des contenus riches en langue française. Sans moindre difficulté ,l'apprenant de FLE peut s'abonner par un clic à ces chaines et reçoit chez lui un apprentissage simple de français. De plus, les enseignants de FLE peuvent adresser les étudiants à chercher sur Youtube des contenus pertinents pour un projet sur lequel, ils travaillent ou pour explorer une de leurs passions.

Nous nous intéressons à la présente recherche à l'apprentissage inversé d'après des chaines de Youtube soit françaises soit francophones qui visent à améliorer la production orale en français. Ces chaines permettent à l'enseignant de publier ou partager des vidéos et des documents sonores. et les apprenants de leur tour ont la possibilité de consulter celles-ci à n'importe quel moment et à n'importe quel lieu.

En classe de FLE, les enseignants de FLE cherchent à rendre leurs apprenants capables de réagir en situations de communication orale afin qu'ils puissent exprimer oralement leurs idées et afin qu'ils puissent contacter entre eux et contacter avec des francophones. Pour ce faire, les apprenants doivent produire des énoncés cohérents et significatifs et ils doivent travailler en classe par rigueur en respectant les règles liées à la prononciation, à l'intonation et au rythme.

En effet la capacité de s'exprimer en situations de communication orale en français reste une véritable 
difficulté aux apprenants de FLE en Egypte . De plus ,le manuel des étudiants de deuxième année secondaire (club @dos plus2) présente un certain nombre de situations d'une manière traditionnelle. Par conséquent le chercheur essaie à la présente recherche de reformuler les situations de communication de la première et la deuxième unités de ce manuel ,sous la forme des capsules vidéo afin d'améliorer quelques compétences de la production orale chez les étudiants du cycle secondaire en téléchargeant ces capsules vidéo sur une chaine de Youtube déjà créée pour qu'ils puissent peuvent consulter le contenu présenté à cette chaine .

Les capsules vidéo présentées à cette chaine sont filmées par le chercheur en coopération avec deux étudiantes de quatrième année du département de français à la faculté de pédagogie. Pour bien profiter du contenu présenté à améliorer la production orale ,selon la stratégie de classe inversée ,le chercheur demandait aux étudiants de deuxième année secondaire de consulter le contenu de cette chaine à la maison et quand ils rentraient en classe, ils coopéraient à exécuter des activités liées à ce contenu.

\section{PROBLEMATIQUE DE LA RECHERCHE}

En dépit de la valeur de l'écrit à l'évaluation sommative des apprenants de FLE en Egypte, l' orale reste très indispensable pour s'exprimer dans les situations de communication. L'oral doit être enseigné pour acquérir aux étudiants de compétences spécifiques qui ne s'acquièrent pas spontanément lors du développement du langage. 
Beaucoup de recherches faites en didactique de FLE ont affirmé que la plupart des étudiants égyptiens inscrits aux départements de français aux facultés de pédagogie ont un niveau insuffisant à l'oral et trouvent des difficultés à se progresser et ils ne sont capables ni de parler, ni de s'exprimer, ni de communiquer dans les différentes situations de communication.

Selon Barthelemy F(2011) «l'apprenant n'a pas l'habilité de s'exprimer oralement dans une langue étrangère et cela peut être perturbé par des facteurs psychologiques comme le manque de confiance en soi et la timidité ou le trac ou tout simplement la peur de se ridiculiser devant autrui.»

Pour bien préciser la problématique de la recherche ,on a appliqué un questionnaire(Annexe 1) à 70 étudiants de deuxième année secondaire. Ce questionnaire se compose de deux parties : la première partie comporte dix items de questions fermées dont les alternances sont (toujours, souvent, quelquefois, jamais) et la deuxième partie comporte une question ouverte de présentation. En analysant les réponses des étudiants, le chercheur a constaté que :

- 90\% des étudiants n'ont pas l'habitude d'échanger la parole en français ni avec leurs collègues ni avec leur enseignant.

- Plus de $97 \%$ des étudiants n'arrivent pas à contacter oralement en français soit face à face soit d'après les réseaux sociaux avec des francophones. 
- Sept étudiants sur dix n'ont pas la possibilité de se présenter oralement en français et ils ne peuvent pas développer oralement un tel sujet.

- Ces indices affirment que les étudiants souffrent de difficultés de la production orale en situations de communications .

\section{QUESTIONS DE RECHERCHE}

- Quelles sont les difficultés principales de la production orale en français desquelles souffrent les étudiants de deuxième année secondaire ?

- Quelles sont les situations de communications principales destinées aux mêmes étudiants ?

- Comment élaborer un programme basé sur l'apprentissage inversé d'après une chaine de Youtube ?

- Quel est l'effet de ce programme sur le développement de la production orale en français en situations de communication auprès les étudiants de deuxième année secondaire ?

\section{HYPOTHESES DE LA RECHERCHE}

- Il y a une différence significative entre la moyenne des notes des étudiants du groupe expérimental et celles des étudiants du groupe contrôle au post test en ce qui concerne la production orale en situations de communication en faveur du groupe expérimental.

- Il y une différence significative entre la moyenne des notes des étudiants du groupe expérimental au pré-test et au post-test, en faveur du post-test en ce qui concerne la production orale en situations de communication. 


\section{METHODOLOGIE DE LA RECHERCHE}

Cette recherche est une recherche quasi expérimentale qui vise à mesurer l'effet d'une variable indépendante l'apprentissage inversé d'après le Youtube sur une variable dépendante qui est le développement de la production orale en situations de communication.

\section{BUTS DE LA RECHERCHE}

Cette recherche vise à:

- Préciser les difficultés principales de la production orale destinées aux étudiants de deuxième année secondaire.

- Déterminer les situations de communications principales destinées aux mêmes étudiants.

- Elaborer un programme basé sur la présentation des capsules vidéos d'après une chaine Youtube à la lueur de la stratégie de l'apprentissage inversé.

- Mesurer l'effet de ce programme sur le développement de la production orale en situations de communication chez les étudiants de deuxième année secondaire.

\section{PERTINENCE DE LA RECHERCHE}

- Affirmer l'efficacité de l'emploi des chaines de You Tube à enseigner le FLE.

- Fortifier l'utilité de la stratégie de l'apprentissage inversé.

- Mettre l'accent sur les difficultés de la production orale en français.

- Guider les enseignants de FLE à employer les chaines de Youtube pour améliorer la production orale en français à la lueur de la stratégie de classe inversée. 
- Mettre le programme de la recherche à la disposition des responsables du ministère de l'enseignement en Egypte afin de le télécharger sur le plateforme des étudiants de cycle secondaire.

\section{OUTILS DE LA RECHERCHE}

Le chercheur a utilisé les outils suivants pour vérifier les résultats de la recherche:

- Une liste de difficultés de la production orale destinée aux étudiants de deuxième année secondaire.

- Une liste des situations de communication orale destinée aux étudiants de deuxième année secondaire.

- Un pré/post test qui mesure le développement de la production orale chez ces étudiants.

- Un programme qui compte neuf modules téléchargés sur une chaine de Youtube créée par le chercheur.

\section{LIMITES DE LA RECHERCHE}

- Le programme élaboré par le chercheur est basé sur la reformulation des leçons de l'unité 1 et 2 du livre club @ dos plus2 en activités langagières.

- La recherche est appliquée aux étudiants de deuxième année au cycle secondaire de l'école (Al Quaisse) au gouvernorat de Minia.

- Le chercheur a expérimenté cette recherche au premier semestre de l'année scolaire 2020-2021.

- Le chercheur a employé la stratégie de l'apprentissage inversé pendant l'application de la recherche.

\section{ECHANTILLON DE LA RECHERCHE}

Le chercheur a choisi l'échantillon de la recherche parmi les étudiants de deuxième année au cycle secondaire 
de l'école d'Al Quaisse (à Minia) Le chercheur a choisi 70 étudiants distribués à deux classes :

- La première classe compte 35 étudiants est le groupe expérimental qui apprennent le programme de l'étude.

- La deuxième classe compte 35 étudiants est le groupe contrôle qui apprennent par la méthode traditionnelle.

- Le chercheur a choisi cette école parce qu'elle est très proche de lui.

\section{TERMINOLOGIES DE LA RECHERCHE Apprentissage inversé}

Selon Marcel Lebrun et Julie Lecoq (2015) « une flipped classroom, ou classe inverse, est une méthode (ou une stratégie ) pédagogique où la partie transmissive de l'enseignement (exposé, consignes, protocole, etc.) se fait à distance en préalable à une séance en présence, notamment à l'aide des technologies (vidéo en ligne du cours ,lecture de documents papier, préparation d'exercice, etc.) et où l'apprentissage fondé les activités et les interactions se fait en présence (échange entre l'enseignant et les apprenants et entre pairs, projet de groupe, activités de laboratoire, séminaire, débat, etc.

Ariane Dumont définit l'apprentissage inversé comme « Approche pédagogique consistant à inverser et à adapter les activités d'apprentissage traditionnellement proposées aux apprenants en utilisant en alternance la formation en classe pour prendre avantage des forces de chacune »

La présente recherche définit l'apprentissage inversé comme une méthode pédagogique qui présente la partie transmissive à distance en téléchargeant des capsules 
vidéos d'après le Youtube tandis que la partie pratique se fait en classe d'après l'interaction entre l'enseignant et les apprenants.

\section{Youtube}

Selon le site de futura sciences, Youtube «est un service en ligne d'hébergement et de diffusion de vidéos en streaming qui intègre des fonctionnalités sociales de partage et de commentaires des contenus. Il s'agit de l'un des sites Web les plus visités au monde.»

La présente recherche définit Youtube comme un média social sur lequel, le chercheur diffuse des capsules vidéos aux étudiants de deuxième année secondaire afin d'améliorer la production orale en situations de communication en français.

\section{Production orale}

Le dictionnaire de Larousse, définit la production comme : « action de produire : fait de se produire ».Manière de se produire.

Hélène Sorez(1995) indique que s'exprimer oralement, «C'est transmettre des messages, généralement aux autres, en utilisant principalement la parole comme moyen de communication ».

La présente recherche définit la production orale comme la capacité des étudiants de cycle secondaire à transmettre des messages oraux comme moyen de communication dans des situations variées.

\section{Communication}

Le dictionnaire Larousse définit la communication comme suit : 
«Action de communiquer avec quelqu'un, d'être en rapport avec autrui, en général par le langage ; échange verbal entre un locuteur et un interlocuteur dont il sollicite une réponse : Le langage, le téléphone sont des moyens de communication.»

La définition de la communication selon le dictionnaire linguistique est «l'échange verbal entre un sujet parlant qui produit un énoncé destiné à un autre sujet parlant, et un interlocuteur dont il sollicite l'écoute et/ou une réponse explicite ou implicite».

La présente recherche définit la communication comme un échange verbal entre les étudiants du cycle secondaire et des interlocuteurs francophones dans des situations variées de la vie quotidienne.

\section{CADRE THEORIQUE}

Le cadre théorique de cette recherche traite les trois axes suivants :

1. La production orale en FLE ;

2. Employer l'apprentissage inversé à enseigner le FLE

3. Le Youtube comme un support en classe de FLE .

\section{Première axe : La production orale en FLE}

CICUREL ,F (2011) voit que la langue française est d'abord et avant tout parlée, elle est un moyen de communication orale qui permet à l'apprenant de français langue étrangère d'intervenir, et d'entretenir avec les locuteurs natifs en mettant l'accent sur des aspects linguistiques (la forme est l'objet des échanges), et en réutilisant de lui-même des structures récemment étudiées. 
A partir du 20ème siècle, la didactique de FLE a suggéré de nouveaux objectifs et des contenus de communication qui visent à enseigner non seulement le français comme langue étrangère mais aussi d'enseigner à communiquer .

La production orale est l'une des principales étapes dans l'acquisition d'une langue .Cependant la majorité d'apprenants sont incapables de communiquer et produire de façon efficace. Asma Melgani (2015) indique que la maîtrise de l'oral est essentielle pour l'étudiant afin qu'il puisse poursuivre son apprentissage dans l'enseignement moyen.

La production orale permet au locuteur de s'exprimer dans diverses situations de communication. Pour pouvoir s'exprimer à l'oral l'apprenant doit avoir des compétences de compréhension et d'expression. L'acquisition d'une telle compétence exige un effort considérable de la part des enseignants et des étudiants qui doivent s'entrainer régulièrement.

La consultation continue des chaines de Youtube qui présentent des activités orales donne aux étudiants la possibilité d'écouter, de répéter et bien évidement d'acquérir les compétences de la production orale.

L'oral est une activité complexe ce qui nécessite aux enseignants les connaissances de ses propres caractéristiques pour une meilleure exploitation. L'oral est caractérisé par son propre métalangage, raccourcis, intonation, forme verbales et répétition. 
Mounira Aouina (2008) présente les caractéristiques de l'oral selon Eberhard Karler comme suit :

1. La prosodie (l'intonation, l'accent, les pauses, ...).

2. Les informations sur le locuteur (voix, état mental, position sociale).

3. L'adaptation de discours du locuteur à son interlocuteur $\gg 7$.

4. L'oral marqué par des pauses, hésitation, reprises, ruptures, le changement de débit,...

5. L'oral fait également l'objet et d'une plus grande implication des locuteurs et auditeurs dans la mesure où ceux-ci font souvent usage d'un style direct, d'autoréférences (moi, je pense que...), d'expressions populaires, d'évidences, d'imprécision...etc

Selon Tagliante (2006), la production orale est construite du fond et de forme:

Le fond est caractérisé par :

- Les idées, les informations que l'on donne, l'argumentation que l'on choisit, les opinions et les sentiments exprimés ; Les illustrations orales, les exemples qui accompagnent les idées ou les informations ;

- -La structuration des idées ; Le langage, la correction linguistique, l'articulation, l'intonation.

- La forme, c'est : L'attitude générale, les gestes, les sourires ;

- La voix, son volume, son débit ; Les regards, les pauses significatives, les silences voulus. » (2006: 82) 
Les apprenants de FLE pratiquent deux types de communication :le premier est officielle ou scolaire qui assure la leçon. Ceci défavorise les échanges spontanés des apprenants, dite formelles et le deuxième est social, cela favorise le libre échange de parole, sans contrainte de thème ou de cadre, dite informelle.

En classe de FLE, la production orale comprend l'écoute, la parole et le langage. Les apprenants en pratiquant la production orale apprennent à partir des informations traitées à déduire les sens des mots utilisés et par conséquent, les maîtriser et les employer ultérieurement pour s'exprimer convenablement.

M Latreche (2015) indique que la production orale en classe de langue étrangère, constitue un véritable moyen des échanges entre les apprenants, puis entre l'enseignant et ses apprenants, elle favorise l'apprentissage et la prise de parole. Pourtant quand il s'agit, pour un apprenant de s'exprimer oralement dans une langue étrangère avec une quantité médiocre du vocabulaire et de la syntaxe, il va fatalement se trouver dans une situation désagréable. Ce malaise, peut le conduire au découragement, à la crainte de prendre la parole, ce qui retardera d'autant son apprentissage.

Pierre-Yves Roux (2003) indique qu'il y'a cinq conditions de l'apprenant pour pouvoir communiquer oralement en français :

1. doit avoir quelque chose à dire : Cela veut dire que l'apprenant doit avoir un sujet captivant ou motivant, 
de son propre vécu ou actuel.

2. doit savoir l'exprimer : Il est très important à l'apprenant d'avoir la liberté de parler ou de s'exprimer même s'il commet des erreurs, il aura recours au mouvement du corps pour l'aider à transmettre l'information.

3. doit avoir le droit de l'exprimer :L'enseignant doit jouer un rôle touchable à faire acquérir à l'apprenant la confiance en soi et l' inciter à prendre la parole et parler, il faut lui faire comprendre qu'il a le droit de s'exprimer.

4. doit avoir envie de parler et de s'exprimer : Cela signifie qu'il doit être motivé par l'enseignant pour s'exprimer et prendre la parole, l'enseignant doit le mettre dans la bonne voix, il doit aussi susciter l'apprenant.

5. doit avoir l'occasion de s'exprimer : L'enseignant doit désigner des types des exercices et des activités orales en qui donne aux apprenants l'occasion de parler. C'est à l'enseignant de faire parler les apprenants.

Pour aider les étudiants de deuxième année secondaire à bien contacter en situation de communication ,le chercheur a analysé deux unités du livre club @dos plus2,il a choisi un certain nombre de situations de communication utiles qui donnent aux étudiants l'occasion de parler librement sans peur.Pendant l'expérimentation, il a encouragé et motivé les étudiants à parler sans les critiquer et sans les choquer. 
Aouissi Youcef(2018) selon l'étude de Oliveira (2010) affirme que les apprenants de FLE souffrent de difficultés en produisant oralement la langue française :

1. Difficultés concernant la grammaire : Les apprenant de FLE ont du mal à appliquer l'ensemble des règles de grammaire qui leur permet de parler correctement la langue française. Parmi les problèmes de grammaire, les problèmes de syntaxe ,d'accord et la construction des phrases.

2. Difficultés concernant la phonétique: En ce qui concerne la phonétique, les apprenants de FLE ont des difficultés à communiquer, à prononcer correctement la langue française.

3. Difficultés concernant conjugaison: Ayant des difficultés à bien conjuguer les verbes, c'est l'un des grands problèmes qui les étudiants confrontent en parlant. Ils ne connaissent pas bien les verbes mais aussi, ils ne savent pas conjuguer les verbes au temps précis. Ils ont également du mal à construire des phrases correctes, qui ont un sens en communiquant en français.

4. 4-Difficultés concernant le vocabulaire: Les apprenants de FLE trouvent de difficultés à bien employer le vocabulaire français, ils apprennent par cœur les mots mais.ils n'arrivent pas à les employer pendant la production orale, mais aussi. De plus, ils ont des doutes sur les mots à utiliser.

A la lueur de difficultés déjà présentées, le chercheur a élaboré une liste de difficultés dont ces étudiants souffrent. Cette liste compte 20 items(Annexe 2) 


\section{Deuxième axe :Employer l'apprentissage inversé à enseigner le FLE}

Khadija Ziane (2017) indique que l'apprentissage inversé ou «classe inversée» représente une grande évolution dans le domaine de l'éducation et une grande attractivité pour les enseignants qui tentent à diversifier et à renouveler leurs expériences, leurs pratiques pédagogiques en classe de FLE avec leurs apprenants . Sans doute,cette stratégie s'est imposée à la didactique de FLE à l'ère du virus Covid-19.

Selon Thomas(2014-2015), la nouvelle génération est très chanceuse de l'apparition de TICE. Il est très facile pour,cette génération qui est bien formée aux compétences numériques d'enseigner le contenu présenté à la lueur de stratégies pédagogiques basées sur l'emploi de TICE .Ainsi que dans le domaine de la didactique de FLE ,il est facile à l'enseignant d'intégrer l'Internet et les TICE dans leur enseignement dans la salle de classe, ces jeunes sont aussi plus conscients qu'il est nécessaire d'apprendre les aptitudes de la communication sociale dans le contexte de la classe.

En fait, l'enseignant peut exploiter les TICE en plusieurs formes de plateformes de diffusion tels que: les blogs, les sites de gestion de classe ,les cites pédagogiques, des logiciels, les réseaux sociaux ( des pages Face book, des chaines sur You tube, et twitter)

L'apprentissage inversé est une méthode d'enseignement dans laquelle la transmission des connaissances se fait dans un environnement personnel et individuel hors de l'école. 
Anna (2020) Montre dans un article publié au site de Easy Lms les avantages de l'apprentissage inversé:

1. Flexibilité D'après l'apprentissage inversé les connaissances sont disponibles en ligne à n'importe quel lieu et à n'importe quel moment . Les apprenants ne sont pas obligés d'assister aux cours directs pour recevoir les informations tandis que le temps de classe est tout consacré à la pratique des activités et des tâches.

2. Personnalisation L'apprentissage inversé permet aux apprenants d'apprendre de manière autorégulée ce qui leur laisse la possibilité de passer plus de temps sur les points qu'ils ne comprennent pas, et moins de temps sur ceux qu'ils maîtrisent déjà.

3. Stimulation d'un apprentissage actif L'apprentissage inversé aide les apprenants à avoir un rôle actif par rapport de l'apprentissage traditionnel, où l'enseignant décide des connaissances à partager et qui s'assure qu'elles ont bien été transmises aux étudiants.

4. Promotion d'un apprentissage pratique L'apprentissage inversé maximise les heures de cours effectifs en présentiel, car cela permet de s'en servir pour mettre en pratique les connaissances déjà acquises à distance.

5. Phillipe Taillard (2014) montre que l'apprentissage inversé est basé sur la capsule vidéo ,cette capsule vidéo est tout simplement, est une séquence vidéo, courte (de 2 à 6 minutes/ ou

6. à10 minutes) et scénarisée, qui précise l'objectif d'un 
cours en particulier, permet d'expliquer des nouvelles notions, donner des renseignements sur un sujet, enrichir des connaissances, ainsi permettant de développer une idée ou un thème.

La présente recherche vise à exploiter des capsules vidéos qui présentent des situations de communication inspirées des leçons de l'unité 1 et 2 du manuel club@dos plus 2. Ces capsules sont filmées par le chercheur en coopération avec deux étudiantes de quatrième année au département de français à faculté de pédagogie de Minia.

\section{Troisième axe : Le Youtube comme un support en classe de FLE}

Cavazza (2015) voit que le terme de médias sociaux numériques désigne un ensemble de services permettant de développer des conversations et des interactions sociales sur internet ou en situation de mobilité. Il classifie les médias sociaux selon leur finalité en quatre types :

1. a) Les médias sociaux numériques de publication qui peuvent se présenter sous la forme de plateformes et du blog ;

2. b) Les médias sociaux numériques de discussion qui peuvent se présenter sous différentes formes ;

3. c) Les médias sociaux numériques de réseautage qui permettent la mise en réseau ;

4. d) Les médias sociaux numériques de partage qui offrent la possibilité aux utilisateurs de partager et de consulter des contenus : vidéos, musiques et photos.

D'après cette classification, Youtube suit le quatrième type de médias sociaux qui permet aux utilisateurs de 
consulter et de publier des vidéos. Debbi et Okaba (2019) trouvent que le Youtube a connu un grand développement aux dernières années ,par ce média social on peut diffuser, partager et publier les informations et les vidéos qui servent l'apprentissage /enseignement des langues.

Le chercheur a choisi ce média social pour être le domaine de sa recherche parce qu'il a les avantages suivants :

1. permet à l'enseignant de publier ou partager des vidéos, des images, des articles, etc., en donnant le lien à ses apprenants pour qu'ils puissent les consulter et réagir par des commentaires ou des discussions entre eux à n'importe quel moment.

2. Les étudiants peuvent surfer l'internet et consulter le contenu présenté sur les chaines de Youtube sans créer un compte et sans avoir un E.mail.

3. 'enseignant peut exploiter Youtube à créer des activités ou des quiz en considérant le niveau de ses apprenants, pour les aider à se développer à leur rythme et savoir le degré de leur progression.

4. favorise l'apprentissage collaboratif; d'après lequel les apprenants s'entraident pour résoudre les situations-problème librement et loin des rituels de la classe.

5. éveille la motivation des étudiants à achever les tâches, dans le domaine de l'apprentissage des langues, et notamment du FLE, les étudiants sont prêts psychiquement et mentalement à faire l'apprentissage. 
6. Ce média social encourage les apprenants ayant des problèmes psychiques (timidité, peur des critiques, le trac,...etc.).

Ayat Ghezal (2011) affirme le même point de vue en montrant qu'il donne aux étudiants l'occasion de poser des questions, adresser des critiques, discuter avec leurs pairs et avoir une opinion qui leur est propre, ce qui agit positivement sur leur progression en matière d'apprentissage

Le You Tube peut être exploité d'une manière récréative en classe de FLE surtout à l'ère de l'épidémie de Covid-19 - En employant ce média social, les enseignants ont la possibilité de présenter du contenu éducatif et des vidéos intéressantes aux apprenants , ce type de médiaux sociaux éveille la motivation des apprenants et les puissent à réaliser de bons résultats. Susan Macneil suggère des idées utiles d'employer Youtube en classe de FLE :

1. L'enseignant visionne une vidéo en classe avec les étudiants en leur demandant de partager leurs observations ou de répondre à des instructions .

2. Avant de commencer une vidéo, l'enseignant a la possibilité de poser une /des question(s) aux étudiants en leur demandant de prendre des notes, puis de publier leurs réponses sur une plateforme web.

3. On peut employer Youtube pour offrir des informations supplémentaires. Il permet aux 
enseignants de fournir aux étudiants de nouvelles informations en présentant à eux des liens vers des contenus intéressants ou en les encourageant à se partager des liens entre eux vers de telles ressources.

4. En suivant la stratégie de classe inversée, l'enseignant peut exploiter Youtube pour livrer aux étudiants le contenu des cours pour le consulter chez eux et quand, ils rentrent en classe, ils exécutent des tâches et font des activités pratiques.

Pour élaborer le programme de la présente recherche ,le chercheur a reformulé les situations de communication des leçons de 1'unité 1et 2 du livre ( club@dos plus 2 ) dans des capsules vidéos filmées par le chercheur en coopération avec deux étudiantes de quatrième année au département de français ;ensuite, le chercheur a téléchargé ces capsules vidéo sur une chaine de Youtube. En appliquant la stratégie de classe inversée, le chercheur a demandé aux étudiants de deuxième année secondaire(membres de l'échantillon de la recherche) de consulter le contenu présenté sur cette chaine à la maison et quand, ils arrivent en classe, il les guident à faire des tâches liées à ce contenu.

\section{CADRE PRATIQUE}

\section{Elaboration des outils}

Le chercheur a élaboré trois outils de la recherche dont l'application vise à répondre aux questions de la recherche et à confirmer les hypothèses déjà mises par le chercheur, ces outils sont : 
1. Premier outil : Une grille de difficultés de la production orale

2. Cette grille vise à préciser les difficultés de la production orale en français chez les étudiants de deuxième année su cycle secondaire en Egypte.

3. Pour élaborer cette grille le chercheur a suivi ces étapes.

4. Le chercheur a d'abord consulté les études qui cherchent l'origine de difficultés de la production orale chez les apprenants de FLE à quelques pays francophones comme l'étude d'Oliviera (2010),l'étude d' Aouissi Youcef (2018),l'étude de M Latreche (2015),l'étude de Sandra et Hanane(2016) et l'étude d' Asma Melgani (2016)

5. a appliqué un exercice de production orale en français niveau DELF A2 tiré d'un site d'internet et il a enregistré la production orale à 20 étudiants à la deuxième année secondaire et il a corrigé cet exercice de production selon un certain nombre de critères.

6. D'après la correction du test de la production orale des étudiants, le chercheur a élaboré une liste des fautes de la production orale que les étudiants de deuxième année commettent cette liste est indiqué au tableau № 1 montre le nombre et le pourcentage des étudiants commettant de chaque faute. 
Tableau № : 1

Liste des fautes de la production orale

\begin{tabular}{|c|c|c|c|}
\hline № & Faute & $\begin{array}{c}\text { Nombre des } \\
\text { commettants la } \\
\text { faute }\end{array}$ & Pourcentage \\
\hline $\mathbf{1}$ & $\begin{array}{l}\text { La prononciation fausse du } \\
\text { vocabulaire acquis. }\end{array}$ & 14 & $70 \%$ \\
\hline 2 & $\begin{array}{l}\text { Ne pas employer le vocabulaire } \\
\text { convenable selon la situation de } \\
\text { communication. }\end{array}$ & 11 & $55 \%$ \\
\hline 3 & $\begin{array}{l}\text { Ne pas prononcer correctement } \\
\text { les mots qui comptent plusieurs } \\
\text { syllabes. }\end{array}$ & 18 & $90 \%$ \\
\hline 4 & $\begin{array}{l}\text { La prononciation fausse les } \\
\text { voyelles et semi-voyelles. }\end{array}$ & 17 & $85 \%$ \\
\hline 5 & $\begin{array}{l}\text { Ne pas prononcer correctement les } \\
\text { lettres nasales }\end{array}$ & 15 & $75 \%$ \\
\hline 6 & $\begin{array}{l}\text { Confondre la prononciation de } \\
\text { lettres : consonnes comme }[p] \text { et } \\
{[\text { b] et }[\mathrm{g}] \text { et }[j] \ldots}\end{array}$ & 13 & $65 \%$ \\
\hline 7 & Ne pas faire la liaison. & 17 & $85 \%$ \\
\hline 8 & $\begin{array}{l}\text { Ne pas produire des phrases } \\
\text { complètes. }\end{array}$ & 19 & $95 \%$ \\
\hline 9 & $\begin{array}{l}\text { Ne pas différencier entre le } \\
\text { masculin et le féminin. }\end{array}$ & 11 & $55 \%$ \\
\hline $\mathbf{1 0}$ & $\begin{array}{l}\text { Ne pas employer oralement les } \\
\text { règles } \\
\text { Grammaticales }\end{array}$ & $\mathbf{1 5}$ & $75 \%$ \\
\hline 11 & $\begin{array}{l}\text { Ne pas employer correctement la } \\
\text { forme verbale convenable. }\end{array}$ & 17 & $85 \%$ \\
\hline 12 & $\begin{array}{l}\text { La prononciation fausse des } \\
\text { verbes conjugués }\end{array}$ & 13 & $65 \%$ \\
\hline 13 & $\begin{array}{l}\text { Construire difficilement des } \\
\text { phrases complexes. }\end{array}$ & 18 & $90 \%$ \\
\hline 14 & $\begin{array}{l}\text { Ne pas faire l'accord de genre et } \\
\text { de nombre. }\end{array}$ & 12 & $60 \%$ \\
\hline 15 & Ne pas articuler la voix. & 9 & $45 \%$ \\
\hline 16 & Ne pas respecter les pauses. & 14 & 70\% \\
\hline 17 & $\begin{array}{l}\text { Ne pas respecter le rythme et } \\
\text { l'intonation. }\end{array}$ & 13 & $65 \%$ \\
\hline 18 & Ne pas argumenter une idée. & 19 & $95 \%$ \\
\hline 19 & $\begin{array}{l}\text { Ne pas enchainer les idées en } \\
\text { parlant. }\end{array}$ & 17 & $85 \%$ \\
\hline 20 & $\begin{array}{l}\text { Ne pas employer les connecteurs } \\
\text { logiques. }\end{array}$ & 16 & $80 \%$ \\
\hline
\end{tabular}


1. 4-Selon cette liste de fautes commises par les étudiants, le chercheur a précisé quelques difficultés de la production orale dont les étudiants souffrent dans une grille compte 20 difficultés classifiées sous cinq composantes concernant le vocabulaire, la phonétique la grammaire, la voix et la structure.

2. 5- Pour vérifier la validité de cette grille, le chercheur l'a proposée à 10 enseignants de FLE au cycle secondaire.

3. 6-Il a élaboré la version finale de cette grille à la lueur des opinions des enseignants (tableau № : 2)

Tableau № : 2

Grille des difficultés de la production orale

\begin{tabular}{|l|l|l|l|}
\hline \multirow{2}{*}{$\mathbf{~}$} & Difficulté & $\begin{array}{l}\text { Nombre des } \\
\text { enseignants /10 }\end{array}$ & Pourcentage \\
\hline 1 & $\begin{array}{l}\text { Difficultés concernant le } \\
\text { vocabulaire : }\end{array}$ & $\mathbf{7 0 \%}$ \\
\cline { 2 - 4 } & $\begin{array}{l}\text { 1-Difficulté de prononcer le } \\
\text { vocabulaire acquis. }\end{array}$ & $\mathbf{5}$ & $\mathbf{5 0 \%}$ \\
\cline { 2 - 3 } & $\begin{array}{l}\text { 2-Difficulté d'employer le } \\
\text { vocabulaire selon la situation } \\
\text { de communication. }\end{array}$ & $\mathbf{7}$ & $\mathbf{7 0 \%}$ \\
\hline $\begin{array}{l}\text { 3-Difficulté de prononcer les } \\
\text { mots qui comptent plusieurs } \\
\text { syllabes. }\end{array}$ & $\mathbf{9}$ & $\mathbf{9 0 \%}$ \\
\hline 2 & $\begin{array}{l}\text { Difficultés concernant la } \\
\text { phonétique. }\end{array}$ & $\mathbf{6 6 \%}$ \\
\hline $\begin{array}{l}\text { 4-Difficulté de prononcer les } \\
\text { voyelles et semi-voyelles. }\end{array}$ & $\mathbf{6}$ & $\mathbf{6 0}$ \\
\hline $\begin{array}{l}\text { 5-Difficulté de prononcer les } \\
\text { lettres nasales. }\end{array}$ & $\mathbf{8}$ & $\mathbf{8 0 \%}$ \\
\hline $\begin{array}{l}\text { 6-Confondre la } \\
\text { prononciation de quelques } \\
\text { lettres consonnes comme [p] } \\
\text { et [b] et [g] et [j]... }\end{array}$ & $\mathbf{4}$ & $\mathbf{4 0 \%}$ \\
\hline
\end{tabular}




\begin{tabular}{|c|c|c|c|}
\hline & 7-Difficulté de faire la liaison & 7 & $70 \%$ \\
\hline & $\begin{array}{l}\text { 8-Difficultés de produire des } \\
\text { phrases complètes. }\end{array}$ & 8 & $80 \%$ \\
\hline 3 & $\begin{array}{l}\text { Difficultés concernant la } \\
\text { grammaire ... }\end{array}$ & & $76 \%$ \\
\hline & $\begin{array}{l}\text { 9-Difficulté de différencier } \\
\text { entre le masculin et le } \\
\text { féminin. }\end{array}$ & 5 & $\mathbf{5 0 \%}$ \\
\hline & $\begin{array}{l}\text { 10-Difficulté d'employer } \\
\text { oralement les règles } \\
\text { grammaticales }\end{array}$ & 6 & $60 \%$ \\
\hline & $\begin{array}{l}\text { 11-Difficulté d'employer la } \\
\text { forme verbale convenable. }\end{array}$ & 8 & $80 \%$ \\
\hline & $\begin{array}{l}\text { 12-Difficulté de prononcer } \\
\text { les verbes conjugués }\end{array}$ & 5 & $50 \%$ \\
\hline & $\begin{array}{l}\text { 13-Difficulté de construire } \\
\text { des phrases complexes }\end{array}$ & 8 & $80 \%$ \\
\hline & $\begin{array}{l}\text { 14-Difficulté de faire } \\
\text { l'accord de genre et de } \\
\text { nombre }\end{array}$ & 6 & $60 \%$ \\
\hline 4 & $\begin{array}{l}\text { Difficultés concernant la } \\
\text { voix. }\end{array}$ & & $66.6 \%$ \\
\hline & $\begin{array}{l}\text { 15-Difficulté d'articuler la } \\
\text { voix. }\end{array}$ & 5 & $50 \%$ \\
\hline & $\begin{array}{l}\text { 16-Difficulté de respecter les } \\
\text { pauses. }\end{array}$ & 7 & $70 \%$ \\
\hline & $\begin{array}{l}\text { 17-Difficulté de respecter le } \\
\text { rythme et l'intonation }\end{array}$ & 8 & $\mathbf{8 0 \%}$ \\
\hline 5 & $\begin{array}{l}\text { Difficultés concernant la } \\
\text { structure et la syntaxe }\end{array}$ & & $80 \%$ \\
\hline & $\begin{array}{l}\text { 18-Difficulté d'argumenter } \\
\text { une idée. }\end{array}$ & 9 & $90 \%$ \\
\hline & $\begin{array}{l}\text { 19-Difficulté d'enchainer les } \\
\text { idées en parlant. }\end{array}$ & 8 & $80 \%$ \\
\hline & $\begin{array}{l}\text { 20-Difficulté d'utiliser les } \\
\text { connecteurs logiques }\end{array}$ & 7 & $70 \%$ \\
\hline
\end{tabular}




\section{Pertinence de la grille}

Cette grille contenant des difficultés de la production orale chez les étudiants de deuxième année secondaire sera mis à la disposition des enseignants et des inspecteurs de FLE en Egypte afin de confronter ces difficultés

\section{2-Deuxième outil : Une liste de situations de communication}

Cette liste contient neuf situations de communication orale tirées du manuel de l'étudiant de deuxième année secondaire.

Pour élaborer cette liste de situations ,le chercheur a suivi les étapes suivantes :

1. a analysé les leçons de l'unité 1 et 2 du livre club @ dos plus 2 que les étudiants de deuxième année ay cycle secondaire étudient en Egypte.

2. a précisé neuf de situations de communication convenables au contenu des leçons de ces unités.

3. Pour vérifier la validité de cette liste, il l'a exposée à 10 enseignants de FLE dont leur opinions sont indiquées au tableau № : 3

4. Selon les opinions des enseignants, il a reformulé la version finale de cette liste Annexe (3)

\section{Troisième outil : Un test de la production orale/ 25}

Le chercheur a élaboré ce test (Annexe 4) afin de mesurer le niveau des étudiants de deuxième année secondaire à la production orale en français. Pour élaborer ce test, le chercheur a consulté un certain nombre de sites 
Tableau № : 2

\begin{tabular}{|c|c|c|c|}
\hline № & Situation & $\begin{array}{c}\text { Nombre des } \\
\text { enseignants } / 10\end{array}$ & pourcentage \\
\hline 1 & La description physique & 9 & $90 \%$ \\
\hline 2 & $\begin{array}{l}\text { Demander et indiquer } \\
\text { un chemin }\end{array}$ & $\mathbf{1 0}$ & $100 \%$ \\
\hline 3 & Décrire mon quartier & 10 & $100 \%$ \\
\hline 4 & Parler des lieux & 8 & $80 \%$ \\
\hline 5 & Parler des professions & 9 & $90 \%$ \\
\hline 6 & $\begin{array}{l}\text { Parler du climat et des } \\
\text { saisons }\end{array}$ & 7 & $70 \%$ \\
\hline 7 & $\begin{array}{l}\text { Parler des nationalités } \\
\text { et des langues }\end{array}$ & 9 & $90 \%$ \\
\hline 8 & $\begin{array}{l}\text { Parler des passions et } \\
\text { des goûts }\end{array}$ & 9 & $90 \%$ \\
\hline 9 & $\begin{array}{l}\text { Parler de la routine } \\
\text { quotidienne }\end{array}$ & 8 & $80 \%$ \\
\hline
\end{tabular}

électroniques qui présentent des modèles du test de DELF niveau A2 puis, il a choisi les types des questions qui correspondent avec le contenu de l'unité 1 et 2 que les étudiants de deuxième année étudient au premier semestre.

\section{La construction du test}

Ce test se compose de trois parties :

\subsection{1- La première partie(entretien dirigé): (5 pts)}

Dans cette partie l'étudiant se présente à l'examinateur en identifiant son nom son prénom, son adresse, ses habitudes ses passions et en parlant de sa famille. L'examinateur pose à l'étudiant des questions supplémentaires au même thème. 


\subsubsection{La deuxième partie (monologue suivi) /10 pts}

L'étudiant choisit un de deux sujets ,il prépare les idées qu'il va traiter sur un brouillon ensuite; il fait une présentation orale du sujet et enfin, l'examinateur pose à l'étudiant des questions complémentaires concernant le même sujet.

\subsubsection{La troisième partie(exercice d'interaction) /10 pts}

Dans cette partie, l'étudiant traite une situation de la vie quotidienne, il joue un dialogue avec l'examinateur. En échangeant le contact avec l'examinateur, l'étudiant doit être capable de :

- Résoudre cette situation ;

- Saluer et utiliser les formes de politesse ;

- Demander et donner des informations.

\subsection{Les critères de correction}

Le chercheur a inspiré d'un manuel de correction de l'examen du DELF un certain nombre de critères pour corriger le test de la production orale publié au site de CIEP qui mesure ces capacités:

1. La capacité de réagir et de répondre très simplement à des questions portant sur son état civil (nom, prénom, nationalité, âge, date de naissance, lieu d'habitation, situation familiale, langues parlées, etc.).

2. La capacité de parler très simplement de ses activités, ses loisirs et/ou ses goûts, et de dire ce qu'il aime faire ou non.

3. L'étudiant est-il capable d'utiliser des formules de 
politesse très simples et de formuler une question très simple?

4. L'étudiant se fait globalement comprendre même si les faux-départs, les pauses et les hésitations peuvent être nombreux. Il peut exprimer son incompréhension et chercher à remédier aux difficultés rencontrées dans la communication par des mimiques, le recours à une langue étrangère ou en sollicitant l'aide de l'interlocuteur?

5. L'étudiant peut-il parler très simplement ?

6. peut décrire très simplement des objets, et parler de manière très simple des différences entre deux images?

7. L'étudiant peut-il comprendre une situation à partir d'une image et exprimer très simplement la nature du besoin?

8. possède des mots isolés ou des expressions mémorisées lui permettant de répondre à des questions simples?

9. L'étudiant tente de produire quelques structures très simples de type " déterminant + nom », « sujet + verbe» et/ou des présentatifs (« c'est...», « il y a...») ?

10. L'étudiant peut -il prononcer un nombre très limité de mots ou d'expressions isolés? 
JRCIET

Vol. 7, No. 3

July 2021

\subsection{Durée du test}

Durée du test $=$ Durée du temps de tous les étudiants

$=$

Nombre des étudiants

$\frac{890}{20}=44.5$ minutes (presque 45 minutes)

\section{3-4.La stabilité du test}

Pour affirmer la stabilité du test, le chercheur a appliqué le test à un échantillon de vingt étudiants de deuxième année secondaire, il a utilisé le programme statistique SPSS à calculer la valeur d'Alpha de Cronbach .77 qui est indiquée dans le tableau №3 et cette valeur montre que le test est stable.

Tableau №3

\begin{tabular}{|cc|c|c|}
\hline & $\mathrm{N}$ & $\%$ \\
\hline Observations & Valide & $\mathbf{2 0}$ & $\mathbf{1 0 0 . 0}$ \\
& Exclue $^{\mathrm{a}}$ & $\mathbf{0}$ & $\mathbf{. 0}$ \\
& Total & $\mathbf{2 0}$ & $\mathbf{1 0 0 . 0}$ \\
\hline
\end{tabular}

Statistiques de fiabilité

\begin{tabular}{|c|c}
\hline Alpha de Cronbach & Nombre d'éléments \\
\hline .77 & 2 \\
\hline
\end{tabular}

\section{3-5.La validité du test}

D'après le la valeur d' d'Alpha de Cronbach indiquée dans le tableau №3, le chercheur a calculé la valeur de la validité comme suit:

La validité $\mathrm{du}$ test $=$

$\sqrt{\text { d'Alpha de Cronbach }}=$ $.77=.88$

Cette valeur de validité affirme que le test est valable. 


\section{ELABORATION DU PROGRAMME DE LA RECHERCHE}

Le programme de la recherche vise à reformuler les situations de communication des leçons de l'unité 1 et $2 \mathrm{du}$ livre club@dos plus 2 sous la forme des capsules vidéos. Ce programme compte neuf modules, pour élaborer le programme de la recherche, on a suivi les étapes suivantes:

1. Analyser les leçons de l'unité 1 et 2 du livre club @dos plus2 afin de préciser les situations de communication présentées dans ces unités.

2. Reformuler ces situations sous la forme des activités langagières.

3. Filmer des capsules vidéo de nouveaux contenu en coopération avec deux étudiantes de quatrième année du département de français.

4. Désigner le cahier des activités de l'étudiants qui compte 9 modules Annexe( 5) en précisant les éléments suivants à chaque module :

- Une introduction de l'importance du programme.

- Les objectifs ;

- Le lexique ;

- Les supports ;

- La stratégie ;

- Le contenu

- L'évaluation .

5. Elaborer le guide de l'enseignant Annexe (6) en précisant

- Une introduction de l'importance du programme.

- Les objectifs ; 
- Le lexique ;

- Les supports ;

- La stratégie ;

- Le déroulement du cours en déterminant le rôle de l'enseignant et le rôle de l'étudiants.

- La correction des exercices.

6. Télécharger les capsules vidéo sur une chaine de Youtube déjà créé par le chercheur .

7. Demander aux étudiants de suivre cette chaine pour écouter le contenu et faire les exercices accompagnés.

\section{Le contenu du programme}

Le programme proposé de cette étude compte 9 modules dont le contenu est tout inspiré du manuel de l'étudiant de deuxième année secondaire, unité 1 et 2 .Le chercheur a reformulé les situations de communication de ces leçons en documents sonores en consultant un certain nombre de sites électroniques et il les a filmées en neuf capsules vidéo à l'aide de deux étudiantes de quatrième année au département de français à la faculté de pédagogie de Minia qui s'appellent Dina et Maurine . Le chercheur a choisi ces étudiantes parmi les étudiants de quatrième année parce qu'il faisait des cours à eux, il a observé qu'elles parlent bien le français et elles ont les compétences de la production orale en français. Après avoir filmé ces vidéo, le chercheur a crée une chaine de Youtube et il y a téléchargé les vidéos en donnant aux étudiants le lien de cette chaine afin de la suivre à la maison. Voici le contenu du programme : 
- Module (1) : La description physique

- Module (2) : Demander et indiquer un chemin

- Module (3) : Décrire mon quartier

- Module (4) : Parler des lieux

- Module (5) : Parler des professions

- Module (6) : Parler du climat et des saisons

- Module (7) : Parler des nationalités et des langues

- Module (8) : Parler des passions et des goûts

- Module (9) : Parler de la routine quotidienne EXPÉRIMENTATION DE LA RECHERCHE

Le chercheur a créé une chaine de Youtube et il a accordé le lien de cette chaine aux étudiants du groupe expérimental pour suivre cette chaine. L'expérimentation du programme de la recherche a duré six semaines pour présenter onze séances distribuées sur deux séances par semaine. Le chercheur a adapté la stratégie de classe inversée.

1. Dans la première séance, le chercheur a présenté aux étudiants l'idée de sa recherche et la raison de la création de cette chaine de You Tube en leur demandant de coopérer avec lui et d'être ponctuels , puis, il a appliqué le pré/test de la production orale de français.

2. A partir de la deuxième séance, les étudiants écoutaient le contenu filmé de chaque module téléchargé sur la chaine de Youtube à la maison et quand ils rentrent en classe, ils faisaient les activités concernant ce contenu. 
3. Au cours de l'onzième séance, le chercheur a appliqué le post test de la production orale de français.

\section{Les problèmes de l'expérimentation.}

1. Un certain nombre des étudiants ont annoncé qu'ils ne peuvent pas comprendre facilement le contenu sonore téléchargé sur la chaine de Youtube et pour résoudre ce problème, le chercheur leur a demandé d'réécouter ce contenu plusieurs fois ou de découper le contenu en plusieurs parties.

2. Quelques étudiants trouvaient des difficultés concernant le lexique, le chercheur leur a donné la possibilité de consulter un dictionnaire électronique.

\section{ANALYSE DES RESULTATS}

L'application de la présente recherche vise à vérifier les deux hypothèses de la recherche.

Pour la première hypothèse :

1-Il y a une différence significative entre la moyenne des notes des étudiants du groupe expérimental et celles des étudiants du groupe contrôle au post test en ce qui concerne la production orale en situations de communication en faveur du groupe expérimental.

Pour vérifier cette hypothèse, le chercheur a appliqué un test de la production orale. En observant les pourcentages des étudiants commettant les fautes de la production orale indiqués au tableau №4, et au schéma № 1 , nous pouvons montrer que le nombre de fautes de la production orale commis par les étudiants du groupe expérimental a été diminué par rapport à celui commis par les étudiants de 
groupe de contrôle au post test. Cela est clair d'après les pourcentages de chaque type de fautes : premièrement ,le pourcentage des étudiants du groupe expérimental qui ont commis les fautes du vocabulaire est $10.4 \%$ tandis que le pourcentage de ceux de groupe de contrôle qui ont commis ces fautes est $59.9 \%$. Deuxièmement, $13.1 \%$ des étudiants $\mathrm{du}$ groupe expérimental ont commis de fautes de la phonétique alors que $81.1 \%$ de étudiants de groupe de contrôle ont commis ce type de fautes. Troisièmement, le pourcentage des étudiants du groupe expérimental commettant les fautes de la grammaire est $21.8 \%$ au moment où le pourcentage des étudiants de groupe de contrôle est $65.7 \%$. Quatrièmement, $20 \%$ des étudiants du groupe expérimental ont commis les fautes de voix tandis que $74.3 \%$ de étudiants de groupe de contrôle ont commis ces fautes. Cinquièmement, $31.4 \%$ des étudiants du groupe expérimental ont commis des fautes de structure et de syntaxe alors que $85.7 \%$ de étudiants de groupe de contrôle ont commis les mêmes fautes.

\section{Tableau №4}

Pourcentage des fautes du groupe expérimental et de groupe de contrôle au post test

\begin{tabular}{|l|l|c|c|}
\hline \multirow{2}{*}{} & Types de fautes & $\begin{array}{l}\text { Pourcentage } \\
\text { des fautes de } \\
\text { G Ex }\end{array}$ & $\begin{array}{l}\text { Pourcentage } \\
\text { des fautes } \\
\text { de G C }\end{array}$ \\
\hline \multirow{2}{*}{1} & $\begin{array}{l}\text { Fautes concernant le } \\
\text { vocabulaire : }\end{array}$ & $\mathbf{1 0 . 4 \%}$ & $\mathbf{5 9 . 9 \%}$ \\
\cline { 2 - 4 } & $\begin{array}{l}\text { 1-Fautes de prononcer le } \\
\text { vocabulaire acquis. }\end{array}$ & $\mathbf{5 7 . 1 \%}$ \\
\cline { 2 - 4 } & $\begin{array}{l}\text { 2- Fautes d'employer le } \\
\text { vocabulaire selon la situation de } \\
\text { communication. }\end{array}$ & $\mathbf{1 1 . 4 \%}$ & $\mathbf{6 2 . 8 \%}$ \\
\cline { 2 - 4 } & $\begin{array}{l}\text { 3- Fautes de prononcer les mots } \\
\text { qui comptent plusieurs syllabes. }\end{array}$ & $11.4 \%$ & $\mathbf{6 0 \%}$ \\
\hline
\end{tabular}




\begin{tabular}{|c|c|c|c|}
\hline 2 & $\begin{array}{l}\text { Fautes concernant la } \\
\text { phonétique. }\end{array}$ & $13.1 \%$ & $81.1 \%$ \\
\hline & $\begin{array}{l}\text { 4- Fautes de prononcer les } \\
\text { voyelles et semi-voyelles. }\end{array}$ & $14.2 \%$ & $88.5 \%$ \\
\hline & $\begin{array}{l}\text { 5- Fautes de prononcer les } \\
\text { lettres nasales. }\end{array}$ & $11.4 \%$ & $77.1 \%$ \\
\hline & $\begin{array}{l}\text { 6- Fautes la prononciation de } \\
\text { quelques lettres consonnes } \\
\text { comme }[p] \text { et }[b] \text { et }[g] \text { et }[j] . . .\end{array}$ & $8.5 \%$ & $71.4 .5 \%$ \\
\hline & 7- Fautes de liaison & $17.1 \%$ & $80 \%$ \\
\hline & $\begin{array}{l}\text { 8- Fautes de produire des } \\
\text { phrases complètes. }\end{array}$ & $14.2 \%$ & $\mathbf{8 8 . 5 \%}$ \\
\hline 3 & $\begin{array}{l}\text { Fautes concernant la } \\
\text { grammaire ... }\end{array}$ & $21.8 \%$ & $65.7 \%$ \\
\hline & $\begin{array}{l}\text { 9- Fautes de différencier entre le } \\
\text { masculin et le féminin. }\end{array}$ & $14.2 \%$ & $51.4 \%$ \\
\hline & $\begin{array}{l}\text { 10- Fautes d'employer } \\
\text { oralement les règles } \\
\text { grammaticales }\end{array}$ & $20 \%$ & $62.9 \%$ \\
\hline & $\begin{array}{l}\text { 11- Fautes d'employer la forme } \\
\text { verbale convenable. }\end{array}$ & $22.3 \%$ & $77.1 \%$ \\
\hline & $\begin{array}{l}\text { 12- Fautes de prononcer les } \\
\text { verbes conjugués }\end{array}$ & $11.4 \%$ & $65.7 \%$ \\
\hline & $\begin{array}{l}\text { 13- Fautes de construire } \\
\text { oralement des phrases } \\
\text { complexes }\end{array}$ & $37.1 \%$ & $80 \%$ \\
\hline & $\begin{array}{l}\text { 14- Fautes de faire l'accord de } \\
\text { genre et de nombre }\end{array}$ & 25.7 & $57.1 \%$ \\
\hline 4 & Fautes concernant la voix. & $20 \%$ & $74.3 \%$ \\
\hline & 15- Fautes d'articuler la voix. & $17.1 \%$ & $77.1 \%$ \\
\hline & $\begin{array}{l}\text { 16- Fautes de respecter les } \\
\text { pauses. }\end{array}$ & $22.9 \%$ & $65.7 \%$ \\
\hline & $\begin{array}{l}\text { 17- Fautes de respecter le } \\
\text { rythme et l'intonation }\end{array}$ & $20 \%$ & $80 \%$ \\
\hline 5 & $\begin{array}{l}\text { Fautes concernant la structure } \\
\text { et la syntaxe }\end{array}$ & $31.4 \%$ & $\mathbf{8 5 . 7 \%}$ \\
\hline & $\begin{array}{l}\text { 18- Fautes d'argumenter une } \\
\text { idée. }\end{array}$ & $28.6 \%$ & $\mathbf{8 5 . 7 \%}$ \\
\hline & $\begin{array}{l}\text { 19- Fautes d'enchainer les idées } \\
\text { en parlant. }\end{array}$ & $31.4 \%$ & $82.9 \%$ \\
\hline & $\begin{array}{l}\text { 20- Fautes d'utiliser les } \\
\text { connecteurs logiques }\end{array}$ & $34.2 \%$ & $88.6 \%$ \\
\hline
\end{tabular}




\section{JRCIET}

Vol. 7, No. 3

July 2021
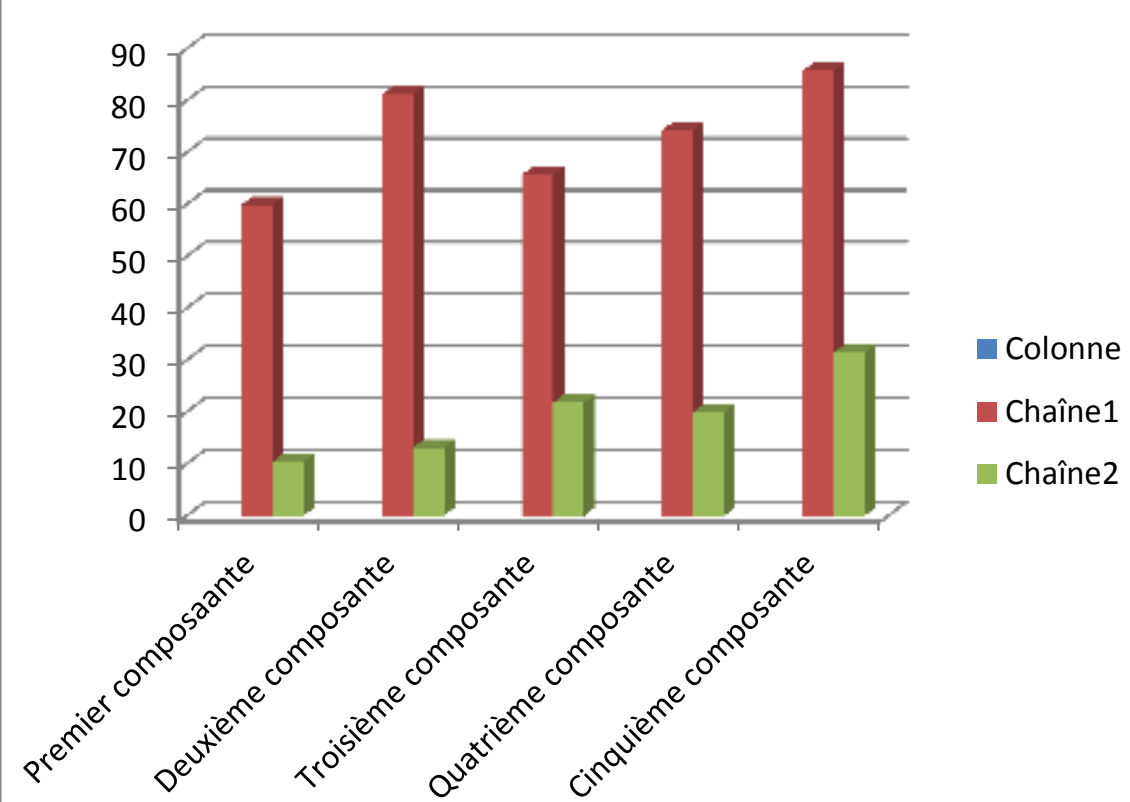

Ces résultats se correspondent avec les résultats obtenus par le programme électronique SPSS indiqués au tableau № 5 .

Tableau № 5

\begin{tabular}{|c|c|c|c|c|c|c|c|}
\hline $\begin{array}{c}\text { Test de la } \\
\text { production orale }\end{array}$ & N & Ma & E & DL & C & T & S \\
\hline Groupe de C & 35 & $\mathbf{1 0 . 2 9}$ & $\mathbf{2 . 1 2 2}$ & 34 & $\cdot$ & 19.75 & $\begin{array}{c}\text { au } \\
\text { niveau } \\
\text { de 0. 01 }\end{array}$ \\
\cline { 4 - 7 } & & $\mathbf{1 8 . 4 3}$ & $\mathbf{2 . 9 2 3}$ & & $\mathbf{5 7 2}$ & & \\
\hline Groupe Ex & &
\end{tabular}

- $\mathrm{N}=$ nombre des étudiants.

- Ma=moyenne arithmétique.

- $\mathrm{E}=$ écart type.

- $\mathrm{DL}=$ degré de liberté.

- $\mathrm{C}=$ Corrélation

- $\mathrm{T}=$ valeur de $(\mathrm{t})$ test.

- $\mathrm{S}=$ significative. 
En observant ces valeurs, on peut montrer que la valeur de moyenne arithmétique des notes des étudiants du groupe contrôle au post test est 10.29 alors que celle-ci du groupe expérimental est 18.43 par rapport à la note totale du test qui est 25. On peut montrer aussi que la valeur de l'écart type des notes des étudiants avant l'apprentissage du programme est 2.12 tandis que cette valeur après l'apprentissage du programme est devenue 2.92. De plus, la valeur de $\mathrm{T}$ Test (19.75) est significative au niveau de.01.

Ces résultats nous conduisent à affirmer que l'emploi du programme de l'étude est utile à améliorer la production orale en situation de communication en français chez les étudiants de deuxième année au cycle secondaire.

\section{Quant à la deuxième hypothèse :}

Il y une différence significative entre la moyenne des notes des étudiants du groupe expérimental au pré-test et au post-test, en faveur du post-test en ce qui concerne la production orale en situations de communication.

Pour vérifier cette hypothèse, le chercheur a analysé les résultats du test de production orale concernant les étudiants du groupe expérimental avant et après l'apprentissage du programme de la recherche. En observant les pourcentages des étudiants qui ont commis les fautes de la production orale indiqués au tableau №6, et 
au schéma № 2 , nous pouvons montrer que le pourcentage des étudiants du groupe expérimental qui ont commis les fautes du vocabulaire avant l'apprentissage du programme est $60.9 \%$ tandis que le pourcentage des étudiants du même groupe qui ont commis ces fautes après l'apprentissage du programme est $10.4 \%$ Pour le deuxième type de fautes concernant la phonétique, nous observons que $80.6 \%$ des étudiants ont commis ce type de fautes au pré test alors que $13.1 \%$ de étudiants ont commis ce type de fautes au post test. Quant au troisièmement type de fautes concernant la grammaire, le pourcentage des étudiants du groupe expérimental commettant les fautes de la grammaire est $67.1 \%$ tandis que le pourcentage des étudiants commettant ces fautes au post test a été diminué à $21.8 \%$. A l'égard de quatrièmement type des fautes concernant la voix, nous trouvons que $74.2 \%$ des étudiants commis ces fautes alors que $20 \%$ de étudiants ont commis les mêmes fautes. Enfin pour les étudiants qui ont commis le cinquième type des fautes concernant la structure et la syntaxe, nous observons que $85.7 \%$ des étudiants ont commis ces fautes au pré test toutefois, ce pourcentage a été diminué à $85.7 \%$ au post test.

Ces indices nous conduisent à constater que le nombre de fautes de la production orale commis par les étudiants du groupe expérimental au post test a été diminué par rapport à celui commis par les mêmes étudiants au pré test. 
Tableau № 6 : Pourcentage des fautes de groupe expérimental

\begin{tabular}{|l|l|c|c|}
\hline$№$ & Types des fautes & $\begin{array}{l}\text { Pourcentage } \\
\text { des fautes de G } \\
\text { Ex Après }\end{array}$ & $\begin{array}{l}\text { Pourcentage } \\
\text { des fautes de G } \\
\text { Ex Avant }\end{array}$ \\
\hline 1 & $\begin{array}{l}\text { Fautes concernant le } \\
\text { vocabulaire : }\end{array}$ & $\mathbf{6 0 . 9 \%}$ \\
\hline $\begin{array}{l}\text { 1-Fautes de prononcer le } \\
\text { vocabulaire acquis. }\end{array}$ & $\mathbf{8 . 5 \%}$ & $\mathbf{6 0 \%}$ \\
\hline $\begin{array}{l}\text { 2- Fautes d'employer le } \\
\text { vocabulaire selon la } \\
\text { situation de } \\
\text { communication. }\end{array}$ & $\mathbf{1 1 . 4 \%}$ & $\mathbf{6 2 . 8 \%}$ \\
\hline $\begin{array}{l}\text { 3- Fautes de prononcer } \\
\text { les mots qui comptent } \\
\text { plusieurs syllabes. }\end{array}$ & $\mathbf{1 1 . 4 \%}$ & $\mathbf{6 0 \%}$ \\
\hline 2 & $\begin{array}{l}\text { Fautes concernant la } \\
\text { phonétique. }\end{array}$ & $\mathbf{1 3 . 1 \%}$ & $\mathbf{8 0 . 6 \%}$ \\
\hline $\begin{array}{l}\text { 4- Fautes de prononcer } \\
\text { les voyelles et semi- } \\
\text { voyelles. }\end{array}$ & $\mathbf{1 4 . 2 \%}$ & $\mathbf{8 2 . 9 \%}$ \\
\hline $\begin{array}{l}\text { 5- Fautes de prononcer } \\
\text { les lettres nasales. }\end{array}$ & $\mathbf{1 1 . 4 \%}$ & $\mathbf{8 5 . 7 \%}$ \\
\hline $\begin{array}{l}\text { 6- Fautes la } \\
\text { prononciation de } \\
\text { quelques lettres } \\
\text { consonnes comme [p] et } \\
\text { [b] et [g] et [j]... }\end{array}$ & $\mathbf{8 . 5 \%}$ & $\mathbf{7 4 . 2 \%}$ \\
\hline $\begin{array}{l}\text { 7- Fautes de liaison } \\
\text { 8- Fautes de produire }\end{array}$ & $\mathbf{1 4 . 2 \%}$ & $\mathbf{6 7 . 1 \%}$ \\
\hline Fautes concernant la & & \\
\hline
\end{tabular}




\begin{tabular}{|c|c|c|c|}
\hline \multicolumn{4}{|c|}{\begin{tabular}{|l|l|} 
& grammaire ... \\
\end{tabular}} \\
\hline & $\begin{array}{l}\text { 9- Fautes de différencier } \\
\text { entre le masculin et le } \\
\text { féminin. }\end{array}$ & $14.2 \%$ & $54.3 \%$ \\
\hline & $\begin{array}{l}\text { 10- Fautes d'employer } \\
\text { oralement les règles } \\
\text { grammaticales }\end{array}$ & $20 \%$ & $62.9 \%$ \\
\hline & $\begin{array}{l}\text { 11- Fautes d'employer la } \\
\text { forme verbale } \\
\text { convenable. }\end{array}$ & $22.3 \%$ & $71.4 \%$ \\
\hline & $\begin{array}{l}\text { 12- Fautes de prononcer } \\
\text { les verbes conjugués }\end{array}$ & $11.4 \%$ & $77.1 \%$ \\
\hline & $\begin{array}{l}\text { 13- Fautes de construire } \\
\text { oralement des phrases } \\
\text { complexes }\end{array}$ & $37.1 \%$ & $80 \%$ \\
\hline & $\begin{array}{l}\text { 14- Fautes de faire } \\
\text { l'accord de genre et de } \\
\text { nombre }\end{array}$ & 25.7 & $57.1 \%$ \\
\hline \multirow[t]{4}{*}{4} & $\begin{array}{l}\text { Fautes concernant la } \\
\text { voix. }\end{array}$ & $20 \%$ & $74.2 \%$ \\
\hline & $\begin{array}{l}\text { 15- Fautes d'articuler la } \\
\text { voix. }\end{array}$ & $17.1 \%$ & $71.4 \%$ \\
\hline & $\begin{array}{l}\text { 16- Fautes de respecter } \\
\text { les pauses. }\end{array}$ & $22.9 \%$ & $74.2 \%$ \\
\hline & $\begin{array}{l}\text { 17- Fautes de respecter } \\
\text { le rythme et l'intonation }\end{array}$ & $20 \%$ & $77.1 \%$ \\
\hline \multirow[t]{4}{*}{5} & $\begin{array}{l}\text { Fautes concernant la } \\
\text { structure et la syntaxe }\end{array}$ & $31.4 \%$ & $85.7 \%$ \\
\hline & $\begin{array}{l}\text { 18- Fautes d'argumenter } \\
\text { une idée. }\end{array}$ & $28.6 \%$ & $85.7 \%$ \\
\hline & $\begin{array}{l}\text { 19- Fautes d'enchainer } \\
\text { les idées en parlant. }\end{array}$ & $31.4 \%$ & $82.9 \%$ \\
\hline & $\begin{array}{l}\text { 20- Fautes d'utiliser les } \\
\text { connecteurs logiques }\end{array}$ & $34.2 \%$ & $88.6 \%$ \\
\hline
\end{tabular}




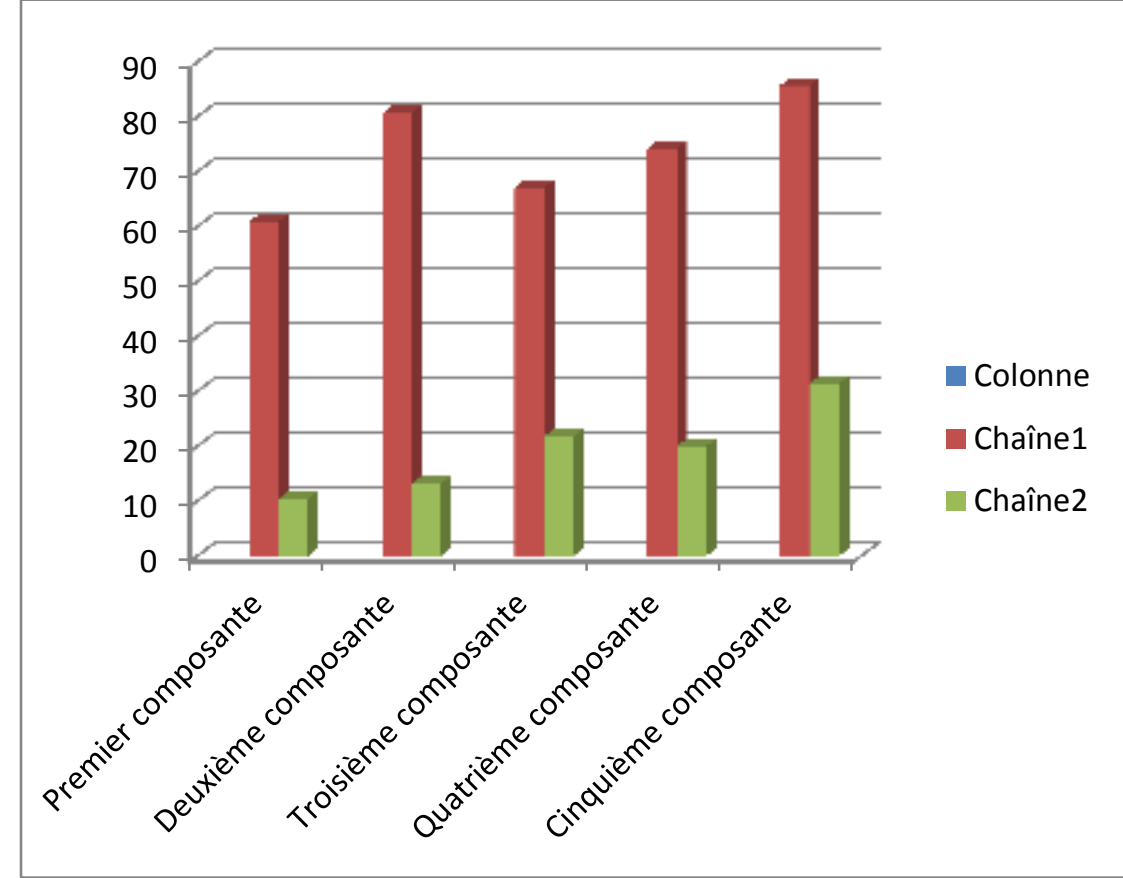

De plus, l'utilisation de programme de SPSS a affirmé les mêmes résultats et cela est clair d'après les valeurs indiquées au tableau № 7.

Tableau № 7.

\begin{tabular}{|c|c|c|c|c|c|c|c|}
\hline $\begin{array}{c}\text { Test de la } \\
\text { production orale } \\
\text { du G. } \\
\text { Expérimental }\end{array}$ & $\mathbf{N}$ & Ma & $\mathbf{E}$ & DL & $\mathbf{C}$ & $\mathbf{T}$ & $\mathbf{S}$ \\
\hline Pré test & \multirow[t]{2}{*}{35} & 10.86 & 1.785 & \multirow[t]{2}{*}{34} & \multirow[t]{2}{*}{.022} & \multirow[t]{2}{*}{12.753} & \multirow{2}{*}{$\begin{array}{c}\text { au } \\
\text { niveau } \\
\text { de } 0 . \\
01\end{array}$} \\
\hline Post test & & 18.43 & 2.923 & & & & \\
\hline
\end{tabular}

En observant les valeurs indiquées dans le tableau, nous pouvons montrer que la valeur de moyenne arithmétique 
des notes des étudiants du groupe expérimental a été élevé de 10.29 au pré test à 18.43 au post test par rapport à la note totale du test qui est 25 . De la même façon, la valeur de l'écart type des notes des étudiants est devenue 2.92 après l'apprentissage du programme tandis que celle-ci valeur avant l'apprentissage du programme était 1.78 . De plus, la valeur de T Test (12.75) est significative au niveau de.01. Ces résultats nous conduisent à affirmer que l'emploi du programme de l'étude est efficace à améliorer la production orale en français chez les étudiants de deuxième année au cycle secondaire.

\section{INTERPRETATION DES RESULTATS}

D'après l'analyse précédente des résultats, on peut constater que le programme de la recherche a atteint son but d'améliorer la production orale en situations de communication en français grâce à l'application de la stratégie de l'apprentissage inversé. L'efficacité de ce programme est due à ces raisons :

1. Les étudiants ont le droit de réécouter plusieurs fois les capsules vidéo téléchargées sur la chaine de Youtube chez eux à n'importe quel moment tandis que l'apprentissage traditionnel en classe ne leur donne pas l'occasion de faire ça.

2. La répétition de l'écoute fortifie la compréhension de l'orale chez ces étudiants.

3. Les étudiants peuvent échanger les publications d'après le Youtube soit avec l'enseignant soit entre eux. 
4. En rentrant en classe, l'enseignant guide les étudiants à produire des énoncés oraux dans des situations de communication, faire les activités et exécuter les tâches en corrigeant les fautes de prononciation ,de grammaire et de style.

Pour la description physique, le premier module a réalisé son objectif de rendre les étudiants capables de décrire physiquement quelqu'un(e). Les étudiants ont écouté chez eux plusieurs fois la capsule vidéo téléchargée sur la chaine de Youtube. Cette capsule vidéo présente un certain nombre d'images des hommes et des femmes et elles indiquent oralement la description physique de ceuxci en montrant l'image puis elle présente un exercice de situations de communication. Quand les étudiants rentrent en classe en faisant la séance directe, le chercheur leur demande de faire la description physique de leur collègues et des membres de famille.

Le deuxième module a atteint son objectif de rendre les étudiants capables de demander et décrire un chemin. Pour ce faire ,les étudiants ont suivi chez eux la capsule vidéo d'après le Youtube. qui présente oralement des modèles des questions pour demander un chemin puis ,elle présente aussi des modèles des phrases pour décrire le chemin en employant les verbes convenables comme aller, tourner ,traverser , avancer, continuer et prendre ensuite ; ils ont écouté de petits dialogues de deux passants qui demandent et indiquent un chemin. Enfin, ils ont fait un exercice de situations de communication. En classe en faisant la séance directe, le chercheur demande à chaque pair des étudiants 
de diriger des petits dialogues pour demander et indiquer le chemin.

D'après le troisième module ,pour être capables de décrire un quartier,les étudiants ont consulté chez eux d'après la capsule vidéo dans laquelle, le chercheur présente oralement des modèles des phrases pour décrire n'importe quel quartier, puis ;ensuite, ils ont écouté un dialogue entre deux personnes qui échangent la description de leurs quartiers. Enfin, ils ont écouté un exercice de situations de communication. En rentrant en classe, le chercheur demande à chaque étudiant de décrire oralement son quartier.

Pour le quatrième module, l'objectif général de ce module est de faire les étudiants savoir les lieux. Pour atteindre cet objectif ,les étudiants ont regardé chez eux la capsule vidéo dans laquelle, le chercheur indique oralement la pertinence de savoir les lieux, puis, ils ont écouté des devinettes qui présentent un certain nombre de lieux. Enfin, ils ont fait un exercice de situations de communication. En rentrant en classe, le chercheur guide les étudiants à exécuter des activités liées aux lieux.

Quant au module № 5 qui a pour objectif de présenter les professions, les étudiants ont consulté plusieurs fois chez eux sur la capsule vidéo qui présente des devinettes pour définir les différents métiers ;ensuite, ils ont fait un exercice de situations de communication. En rentrant en classe, le chercheur guide les étudiants à exécuter des 
activités liées aux métiers et il leur demande d'indiquer les métiers de leurs parents.

Le sixième module vise à rendre les étudiants capables de décrire le climat à n'importe quel lieu et à n'importe quelle saison. Pour atteindre cet objectif ,les étudiants ont regardé la capsule vidéo qui présente oralement des modèles des phrases accompagnées des images pour décrire le temps en différentes saisons ; ensuite, ont écouté un bulletin météo . Enfin, ont fait un exercice de situations de communication. En rentrant en classe, le chercheur guide les étudiants à exécuter des activités liées au climat et il demande à un certain nombre d'étudiants de jouer le rôle d'un météorologiste en présentant une météo.

Le module № 7 a réalisé son objectif de rendre les étudiants capables d'exprimer les adjectifs de nationalité ,la ville capitale de tel ou tel pays et les langues parlées à n'importe quel pays . Pour ce faire ,les étudiants ont suivi la capsule vidéo dans laquelle, le chercheur présente les adjectifs de nationalité, les langues et les villes capitales d'un certain nombre des pays aux différents continents, puis, ils ont écouté un dialogue entre un touriste et un guide. Enfin, ils ont fait un exercice de situations de communication. En rentrant en classe, le chercheur guide les étudiants à exécuter des activités liées aux langues et aux adjectifs de nationalité et il leur demande d'exprimer la nationalité des personnages célèbres.

Pour réaliser l'objectif du huitième module de rendre les étudiants capables d'exprimer leurs passions et leurs goûts 
,les étudiants ont regardé la capsule vidéo qui présente des modèles des phrases pour exprimer les passions et les goûts puis, ils ont écouté les passions et les hobbies de quatre personnes, ensuite; ils ont fait un exercice de situations de communication. En rentrant en classe, le chercheur guide les étudiants à exécuter des activités de ce module.

Pour le dernier module : qui a pour objectif de rendre les étudiants être capables d'exprimer les activités quotidiennes de quelqu'un, les étudiants ont suivi la capsule vidéo dans laquelle, le chercheur présente modèles des phrases qui expriment l'agenda quotidien de quelqu'un, puis, ils ont écouté deux modèles différents des activités quotidiennes . Enfin, ils ont exécuté un exercice de situations de communication. En rentrant en classe, le chercheur guide les étudiants à exécuter des activités liées au sujet et il leur demande d'annoncer l'agenda du jour de leur père.

\section{RECOMMANDATIONS}

D'après les résultats de la présente recherche, le chercheur recommande que :

- Les enseignants de FLE au cycle secondaire s'intéressent à développer la production orale chez leurs apprenants.

- Les enseignants de FLE créent des chaines de You Tube pour y télécharger des contenus oraux en français.

- Les responsables de l'enseignement de FLE téléchargent le programme de cette recherche sur le plateforme du 
ministère de l'enseignement en Egypte pour être à la disposition des étudiants de cycle secondaire.

- Les apprenants de FLE en Egypte suivent les chaines de You Tube qui présentent des documents sonores en français.

\section{ETUDES SUGGEREES}

- Etudier l'effet de l'emploi des chaines de You tube sur le développement de la compréhension orale en français.

- Analyser le contenu du livre de club @dos plus à la lueur de l'approche communicative.

- Employer la stratégie de l'apprentissage inversé pour améliorer la communication écrite en français au cycle secondaire.

\section{BIIBLIGRAPHIE}

- Abdelaziz M L : L'expression orale: Pratiques et difficultés en classe de FLE,Cas des étudiabts de le 1ère année LMD français, Mémoire du diplôme du master ,Faculté des lettres et des langues ,Université Hama Lakhadar El-iued,Algérie ,2014-2015 (p 30-31)

- Ahmed D, Ali O (2019):L'apport des chaines YouTube dans l'apprentissage d'une compétence grammaticale.Le cas des étudiants de 2ème année français de l'université Echahid Hamma Lakhdar - El-Oued,mémoire de diplôme de master,2019,p :24.

○ Anna(2020):https://www.easy-lms.com/fr/base-connaissances/apropos-des-classes-inversees/ avantages-inconvenientsapprentissage-inverse/item 10610

- Aouina, Mounira: L'enseignement/apprentissage de la compréhension orale le biais d'un document sonore, mémoire de magistère, université de Batna, 2007-2008, p20. 
○ Aouissi Youcef(2018) :Les origines des difficultés à l'oral chez les étudiants de FLE.Memoire de diplôme de Master,Faculté des langues et des lettres,Université du 08 mai 1945, Guelma, Algérie,2017-2018(p.18)

- Ariane D, Denis B : La pédagogie inversée enseigner autrement dans le supérieur avec la classe inversée, 2016.p39.

- Asma Melgani : Les difficultés de la production orale chez les apprenants de la 2ème année moyenne, Mémoire pour l'obtention du diplôme de master ,faculté des lettres et des langues étrangers, université mohamed khider - biskra ,Algérie 2015-2016,p 64,

- Ayat G, S (2011). Problématique de l'enseignement-apprentissage de la lecture/écriture à l'ère du multimédia, Université de Mostaganem.p : (64-65).

- Barthelemy F, Groux D \& Porcher L, Français langue étrangère, Harmattan, 2011,(p 16)

- Cavazza Fred :Panorama des médias sociaux en 2015

- Cicurel , F : Les interaction dans l'enseignement des langues, Ed Didier, Paris , 2011, P12.

○ Cregut, Thomas:Rôle et usage des TICE dans la pédagogie inversée. Collège le moulin A vent, Thorigny-sur-Marne, mémoire de Master MEEF, sous la direction de SCHWER Sylviane, Académie de Créteil p02-03.2014-2015.

○ Dictionnaire de Larousse, La présente, France , 2008 , P339. Dictionnaire du Robert,;Paris,2005, P340.

- Duboit, J.G, Mathé, dictionnaire de linguistique et des sciences du langage, 1973.

○ Eberhard, Karler, Universitat , http:// homepages , Uni tubingen.de . Le Robert, illustre, la production : «action de provoquer un phénomène ». 
○ Khadidja Ziane :Développer autrement la compétences de la production orale par le biais de la classe inversée,Cas des étudiants de 3ème année de licence de français, mémoire du master académique ,Université de Mohamed Boudiaf, M'sila, 2017.

o Marc Zaffagani: You Tube: Qu'est-ce que ... : c'est: https://www.futura-sciences.com/tech/definitions/internetyoutube- 16495/le7janvier 2021

- Marcel L, Julie L : classes inversées, enseigner et apprendre à l'endroit, Réseau CANOPÉ.2015.p16.

- Oliveira ,D ,N : Les difficultés des élèves à communiquer en français à l'oral,en classe de 9ème et au 10ème au lycée Abilio Duarte de Palmarejo : réalités et perspectives Maitrise en Etude françaises, Université du CAP VERT, 2010,p12.

o Phillipe Taillard: "En classe inversée Ayez de la ressource" article $\mathrm{n}^{\circ} \quad 72$,publié Septembre-Octobre 2014 en "TECHNOLOGIE",

O Pierre Y R (2003) : L'oral en classe de langue : de la production à l'ex pression, Le Français dans le monde $n^{\circ} 327$, juin 2003,p : 3638.

- Sandra D,Hanane H: Les difficultés de l'enseignement /apprentissage de l'oral en classe de FLE : Le cas des élèves de la 4éme année moyenne, mémoire de diplôme du master II,Faculté des lettres et des langues, Université de Mouloud Mammeri Detizi-Ouzou, Algérie,2016,p(45-50)

○ Sorez, Halène : Prendre la parole,Hatier1995,p5.

- Susan Macneil : Des idées pour utiliser YouTube en classe

○ file://D:/Youtube/Des\%20id\%C3\%A9es\%20pour\%20utiliser\%20 YouTube\%20en\%20classe\%20_\%20Profweb.html 
- Tagliante,C. (2006) : La classe de langue. Paris, Nouvelle édition, CLE international.

\section{Sitographie}

○ https://www.larousse.fr/dictionnaires/francais/ communication/17561

○ http://www.fredcavazza.net/2015/05/29/panorama-des-mediassociaux-8/1/2021.

○ https://www3.ciep.fr/LF/DILF/GestionSessions /docs/manuel_correcteur.pdf

○ https://www.victorias.fr/dossiers/cours-anglaisdictionnaire/apprentissage-inverse.html

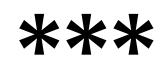

